\title{
Study on Accessible Long-Term Care Mobile Application for Visually Impaired Users
}

\author{
Kan C. W. Russ \\ Innovative DigiTech-Enabled Applications \& Services Institute, Institute for Information Industry, Taipei City, Taiwan
}

\begin{abstract}
A result revealed by the Department of Statistics, 29,000 elderly people have registered for Visually Impaired Card in 2014, which was $51.2 \%$ of all visually impaired people. With annual growth rate of $1.56 \%$, this number increased yearly by 1,100 people. According to WHO, 285 million people worldwide were estimated to be visually impaired, which is $4.24 \%$ of overall population in 2012. From age distribution point of view, most visually impaired people, accounted for $2.76 \%$ of overall population, were above age 50. In average, there are 7 visually impaired people in every 100 people above 50 years old. In Taiwan, 549,000 people over age 50 are estimated to be visually impaired. Therefore we expect there is a large amount of visually impaired people. The main purpose of this research is to collect adaptability information on the cognitive model of senior visually impaired people on their work status, social participation status, and leisure activities via questionnaire survey. Furthermore, descriptive video service is used to as an accessible long-term care application for visually impaired senior users. To solve disability circumstances and improve home care quality through visually impaired APP POC (proof-of-concept). The result of this research will serve as an important basis for other researches in related study field, a reference for practice application. As a result in encouraging profit-seeking enterprises design a user oriented products. And even be an opening for mobile accessibility services benchmark on technology social care for disability and senior users.
\end{abstract}

Key words: Mobile computing, mobile accessibility, UX design, CareTech, visually impaired users.

\section{Subject and Purpose}

In 2014, according to the Department of Statistics, acquired visual-impairment accounted for $88.3 \%$ of the estimated 60,000 people living with visually impaired. There are 7,840,000 people aged 50 and over, and among them, there are 549,000 people suffering from visually impaired.

Thirty visually impaired professionals were invited to solve visually impaired scenario with big data implementation and its extended services. These professionals include visually impaired people (congenital visual-impairment, and acquired visual-impairment) and their support groups (including oculists, family members of eye diseases patients, staff, social workers, and volunteers at social

Corresponding author: Kan C. W. Russ, Ph.D., product manager, research fields: CareTech, assistive technology, mobile App accessibility, mobile applications best practices for user with disabilities, accessible web-design, usability for aging user $\&$ user with special needs, system automation. welfare organizations, special education centers, etc.).

In this research, we collected and analyzed disability situations visually impaired people encountered with food, clothing, housing, transportation, education, entertainment, medicals, and working, at home environment and public spaces.

Apps for blind and visually impaired now on the market are considered hard or cannot be used by many visually impaired people, resulted in low market penetration. Therefore, we would like to find out what the service is lack of based on user experience. This research is based on users' daily life experience, with very thorough and detailed observation and interviews to find out usabilities and accessibilities, the essentials of service and development, for the service provider and developer.

\section{Research Purpose}

The purpose of this research is to provide suggestion on improving information display, function 
combination, voice feedback, and user interface of mobile accessibility app services. To solve the difficulties on user operations, this research analyzed some powerful voice recognition systems and then developed keyboard shortcuts which require very easy memorizing, intuitive Multi-Touch gestures, and fully functionalized voice-user interface. With a creative mind, we developed a mobile device app based on early stage research framework to provide more recognition services to improve the living environment and self-care ability of individuals with disabilities. For example, visually impaired females have needs on doing makeup and dressing; visually impaired people have needs on recognizing expiration date or pharmaceutical compositions; non-electronic text, etc. [1].

\section{Research Methods}

When interviewing professionals, Quantitative Research was used on questionnaires pretest and Qualitative Research was used on in-depth interviewing posttest. Firstly, we have to define Qualitative Research. According to 鄭陸霖, the ex-Associate Research Fellow at Institute of Sociology, Academia Sinica, the most proper definition of Qualitative Research simply defined it as "non-qualitative". Qualitative in-depth interviewing emphasizes on the process of participatory sense-making. In-depth interviewing is an interactive process but not pouring out one's thoughts and emotion unilaterally $[5,6]$.

Main reasons why Qualitative Research was chosen to be the research method are as following:

- Understanding the specific subject, group, or incident is more important than the statistic objective result.

- Practical concerns like limited time, manpower and budget make it difficult to do large scale questionnaire survey.

- Number of specimens was limited because the research subject was focused on some specific unique cases.

Visually impaired people are the subject this research studies at. Base on their needs, which are much more important than pure statistical data, the product characteristic and the developing direction can be decided. Usually it is very hard to reach visually impaired people. Therefore, rather than doing large scale questionnaire survey through social welfare organizations, close-ended questionnaire with quantitative data analysis plus Qualitative Research on open-ended questionnaire is more likely to get efficient answers on problems. Furthermore, this questionnaire survey also targeted visually impaired people support groups, like social workers, family members, oculists, special education center staff, etc. By doing this, it would make this questionnaire be diverse, complete, subjective and objective.

It is more difficult to do in-depth interview using Qualitative Research than Quantitative Study, because Qualitative Research is not a structural process. Furthermore, when interviewing, the researcher cannot keep neutral but with a subjective idea. It is not that the researcher is doing this on purpose but because it is unavoidable to add subjective thoughts and judgements. Sensitivity is also very important when collecting valuable information.

There are many papers, whether official or unofficial ones, are not accessible. That makes in-depth interviewing extra important, because it helps researchers understand unshown principles and key points in many institutions or groups. Before doing in-depth interviewing, researchers have to study related documents, industry reports, news reports, and other related researches to understand that group or the history line of a certain incident. Also, researchers can add those key issues which were missing in the above data into the in-depth interview.

Base on the above reasons and the characteristics of Qualitative Research, it is expected to bring more benefits to the early stage research of this project. On this basis, open the conversation between technology 
and visually impaired people.

In this research, 200 questionnaires were issued and 182 were valid. Besides, there are 30 in-depth interviews. Interviewees include support system professionals (health care system and social workers), congenital and acquired visual-impairment with equal proportions.

\section{Users Background Demographic}

\subsection{Questionnaire Respondents Background} Demographic

(1) Support System

Support system mainly refers to those groups which provide supports in visually impaired people's daily life. These groups include healthcare workers, social workers, education resources (mobility specialist, special education classrooms, etc.), family members, partners, friends. They accompany, listen, and react with visually impaired people. Especially family members, partners, and friends get along with visually impaired people in a long period of time. That makes them be able to tell accompanying difficulties with empathy. Therefore, from questionnaires, we can find out visually impaired people's needs in the angle of these companions [3, 4].

(2) Congenital Visual-Impairment

In this questionnaire, we divided Congenital Visual-Impairment into three categories, congenital, congenital low vision then gradually lost vision, and congenital low vision. Visual perception differences between these three categories are that, congenital visual-impairment does not have vision perception, and the other two have some vision perception. People who are Congenital Visual-Impairment have better facial vision when the environment changes.

(3) Acquired Visual-Impairment

Acquired Visual-Impairment can be divided into three categories according to causes: accidents, rare diseases, or general diseases. People who are Acquired Visual-Impairment have had normal vision perception before they became visually impaired.
Because they have vision perception, compared to those who are Congenital Visual-Impairment, they have weaker facial vision when the environment changes. Therefore trainings and habits are needed to enforce and improve facial vision.

\subsection{Cognitive Differences in Daily Life Tasks}

We divide daily life tasks into 8 categories:

(1) Food: dining and food selection;

(2) Clothing: shopping for clothes and collecting style;

(3) Housing: housing surrounding, environment, and space allocation;

(4) Transportation: use public transport;

(5) Education: school education and further study;

(6) Entertainment: including audio book, radio, and video;

(7) Medicals: hospital traffic flow, procedure, and support system;

(8) Others: anything that does not below to the above 7 categories.

The common point between the above 8 categories is the need for allocation and mobility. No matter the mobility refers to a person or goods, it all related to the dynamic character and interactivity between the person and goods. Since mobility is a very important daily life task for visually impaired people, we derived the discussion from this to needs in the above 8 daily life tasks. There are differences in cognition between subjective needs and objective supports. Thus, needs of people with disabilities are supported by support systems. The incoordination and inconsistence between subject and object results in many accessibility resources are laid idle. In other words, there is lack of real accessibility resources. What are those actual differences in cognition is the problem this research discusses.

\section{Results and Discussion}

Based on Quantitative Analysis, Fig. 1 displays quantitative indicators of needs of people with disabilities. 


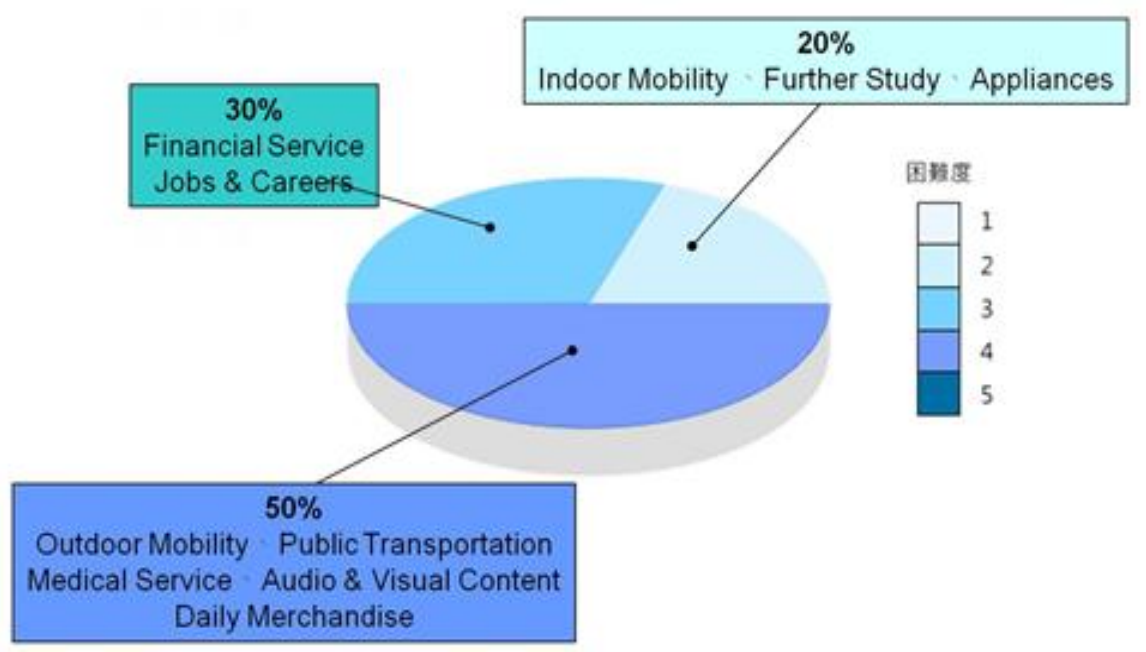

Fig. 1 Sratistical overview-social engatement.

Fifty percent of interviewees consider outdoor mobility, public transportation, medical service, audio $\&$ visual content, and daily merchandise difficulty 4 . Thirty percent of interviewees consider financial service and jobs \& careers difficulty 3. Twenty percent of interviewees consider indoor mobility, further study, and appliances difficulty 2.

In this difficulty index, it is hard to evaluate if we do not separate interviewees into groups. In order to clarify whether there exist cognitive differences in daily life tasks between Support System and Visual-Impairment, we classify interviewees into three groups which are Support System, Congenital Visual-Impairment and Acquired Visual-Impairment.

People with congenital visual-impairment do not have 2D graphic concepts. Therefore, they find most difficult on financial service, audio \& visual content, daily merchandise, and jobs \& careers where text and printed information are greatly used. In financial service like opening an account, people have to do many signatures due to laws of privacy. People with congenital visual-impairment are not capable of writing and therefore they need other people's help. Most audio \& visual contents are displayed on the screen or printed media where visual understanding on pictures and words are needed. When doing daily merchandise, it is hard to recognize the item information like ingredients and expiration date with congenital visual-impairment. As for jobs \& careers, due to natural limit, they cannot process information which needs much reading. Plus it is more difficult and time consuming for them to move than sighted people, therefore accessible facilities are required in the working environment which makes cost increases for the employer. That reduces the willingness for the employer to hire visually impaired people and results in very restricted job choice for them.

There is a very high repeatability between acquired and congenital visual-impairment when speaking of difficulty index. However if we look closer, the difficulty of mobility is one level higher for people with acquired visual-impairment. This is an indirect proof of facial vision which was mentioned in Users Background Demographic. People with congenital visual-impairment have better facial vision than people with acquired visual-impairment. Therefore, people with congenital visual-impairment rank this difficulty index in the middle. As for people with acquired visual-impairment, they normally have to spend 3 to 8 or longer years, depending on people, to practice and adapt to positioning. Facial vision, on the other hand, affects braille learning and consequently people with congenital visual-impairment are faster on learning braille through finger touches. As for people 
with acquired visual-impairment, they can only be more effective in learning braille after being adapted to facial vision. In average, it is not efficient or effective for people with acquired visual-impairment to learn braille because of lower facial vision.

In contrary, people with acquired visual-impairment usually have 2D graphic concepts. With other people's help on paper mobility, they normally can accomplish writing tasks. Therefore, the difficulty level on signature and writing at financial service is lower for them. However, the law problem on bank privacy policy still exists. As for medical service, since the dynamic character of them are worse than sighted people, when the hospital is short of support systems like volunteers, they have to count on hospital volunteers, their family or friends. Therefore, they normally have to spend hours on the medical process from going to the hospital, making appointment, waiting for the number is called, seeing the doctor, paying and getting medicine, until leaving the hospital.

We got a conclusion based on those needs classified as highly difficult for people with visual impairment. Thus, because of the unfriendly environment, they cannot expert their innate abilities like mobility and decision making. Unfriendly environment is especially obvious for people with visual impairment when going to the doctor, taking the bus, and withdrawing money, which sighted people are very used to. There is no screen reader or other sufficient real-time notification at bus stops, so people with visual impairment cannot get the bus status; nor does the bus driver know if there are any people who want to get in. As for ATM machines, the problem was caused by lacking of standard specification at accessibility voice instruction. Some are with touchscreen and some are with keyboard; some voice instructions only instruct halfway and some voice instructions only start after people's operation; the place to withdraw money is different on every ATM; For many ATMs, there is no headphone plug, so people with visual impairment cannot hear detailed response which makes them not know if they are touching the correct place and results in much higher safety risks on withdrawing money than sighted people. To sum up, even many ATMs have accessibility voice instruction; it often does not work for people with visual impairment.

To summarize the questionnaire responses from people with congenital and acquired visual impairment, we can find out their needs from the resistance and power on their daily life. The visual impression before people lose their sight can be a power or a resistance. For people with acquired visual impairment, having color and Chinese character concepts can be a power because it helps them on learning communication by referencing related experiences and know-how; but it can also be a resistance when learning braille because they do not have enough facial vision. And vice versa, for people with congenital visual impairment, facial vision is their power when learning braille, but lack of 2D graphic concepts is a resistance on learning texts. Signature, for instance, is a very difficult task for them [2].

Blind spots often exit in support systems for visually impaired people in all professions, because they tend to imagine instead of truly understanding visually impaired people's need on the service and design. Fig. 4 is the support system data analysis on healthcare workers, mobility specialists, and support systems like visually impaired people's partner. We can find out blind spots when we compare items scored as difficulty levels 2, 3, and 4 on Figs. 2-4. Twenty percent of support system interviewees scored outdoor mobility as difficulty level 4 while people with congenital visual impairment only scored it as difficulty level 3; Sixty percent of support system interviewees scored audio \& visual content and jobs \& careers as difficulty level 2 while people with congenital and acquired visual impairment both scored it as difficulty level 4. 


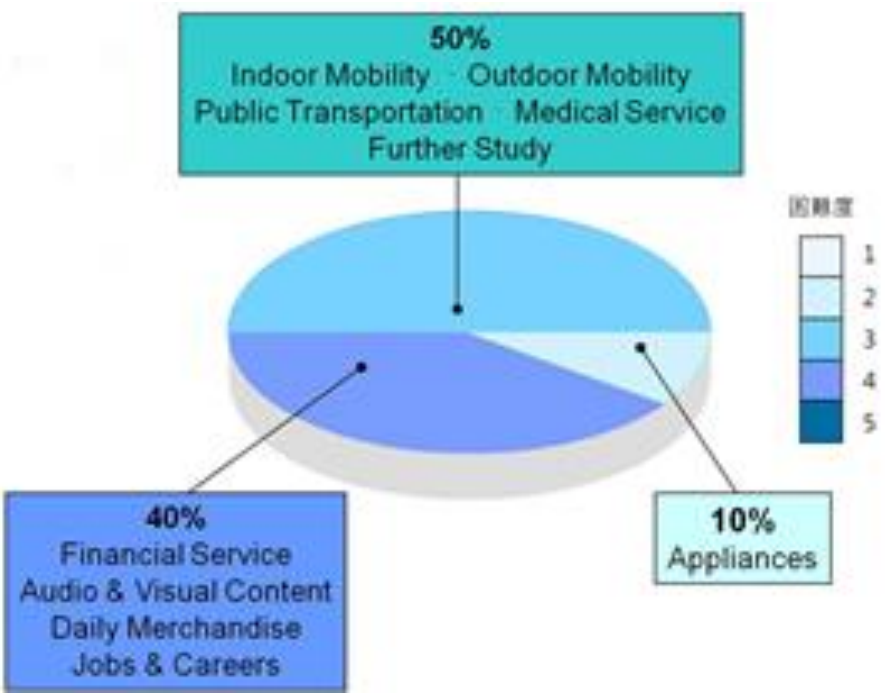

Fig. 2 Statistical analysis of congenital visual-impairment.

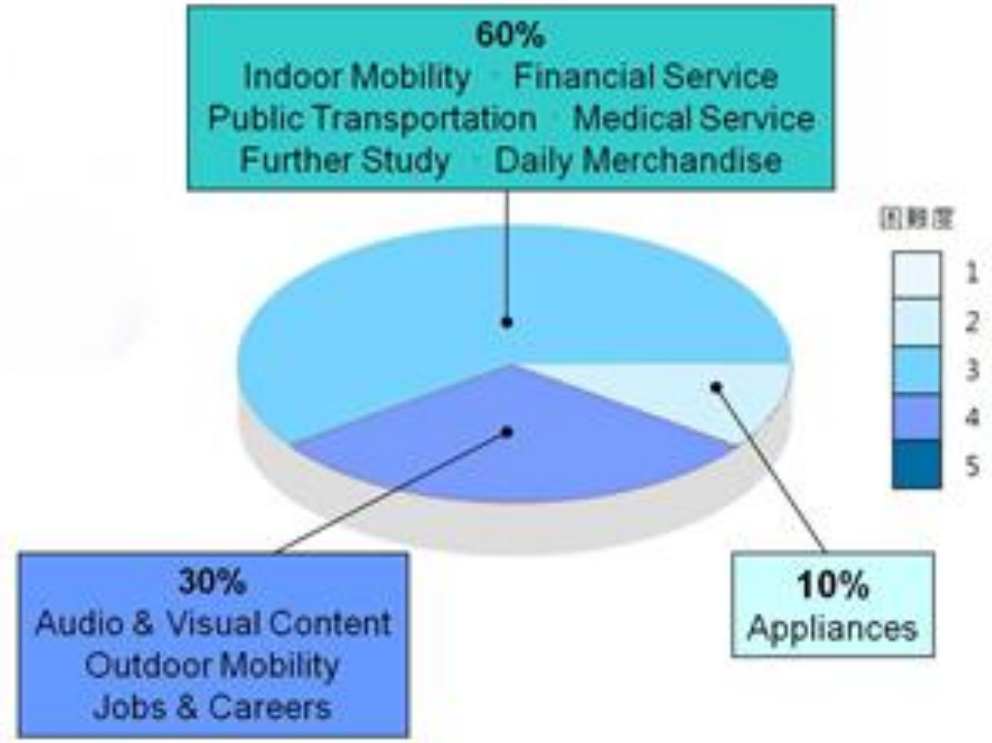

Fig. 3 Statistical analysis of acquired visual-impairment.

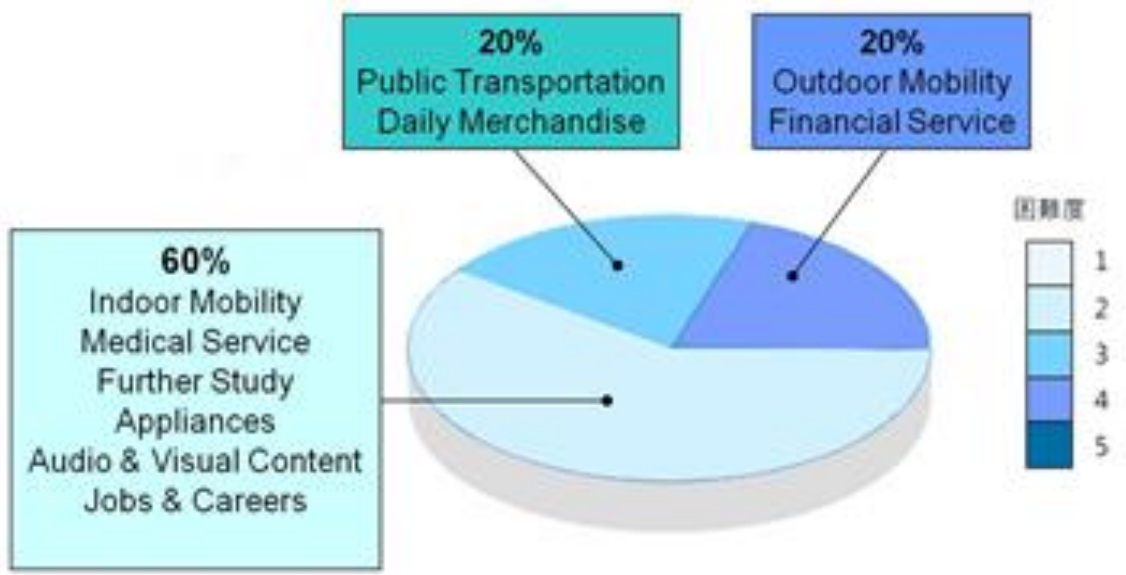

Fig. 4 Statistical analysis of support system. 
If we subdivide support system into non-professional (family, friends, partners) and profession ones, it is very possible to find out that the differences on support involvement level and position lead to different cognition on difficulty level. However, since there is not enough number of support system samples, we did not subdivide to avoid error.

\section{Conclusion}

Fig. 5 displays the quantitative data of important but non-urgent needs. In indoor fields, it is suggested to rely on software (support system) while technology subsidiary. They complement each other to make sure there is no immediate accident. Furthermore, important but non-urgent needs should be discussed in later stage due to limited resources. Please refer to Fig. 5 below.

Speaking of public transportation, the reason why taking MRT or HSR (high speed rail) is less problematic is that their software support is more mature. Their staffs have been trained for professional support system, so they have better understandings on how to provide appropriate support on the mobility needs of visually impaired people. In the contrary, staffs of railways and buses were not well trained on accessibility guidance, so visually impaired people cannot take these two transportations by themselves. To solve the accessibility problem on public transportation, software can be enhanced by communicating with related units to strengthen staff trainings on support system. Technology can complement software when it is not enough to support. For instance, on passenger side, technology can be applied on screen reading and real-time bus notification at bus stops; as on bus drivers side, technology can be applied on real-time passenger information notification. However, since this item is important but non-urgent need, it can be discussed in later stage of this research.

Fig. 6 is the intersection of important and urgent needs between people with congenital or acquired visual impairment and support systems. Both people with congenital visual impairment and support systems consider financial service important and urgent. As it is mentioned above, congenital visual impairment causes difficulties on writing, plus financial law makes it even harder to do even the most basic opening account. Not to mention other financial service.

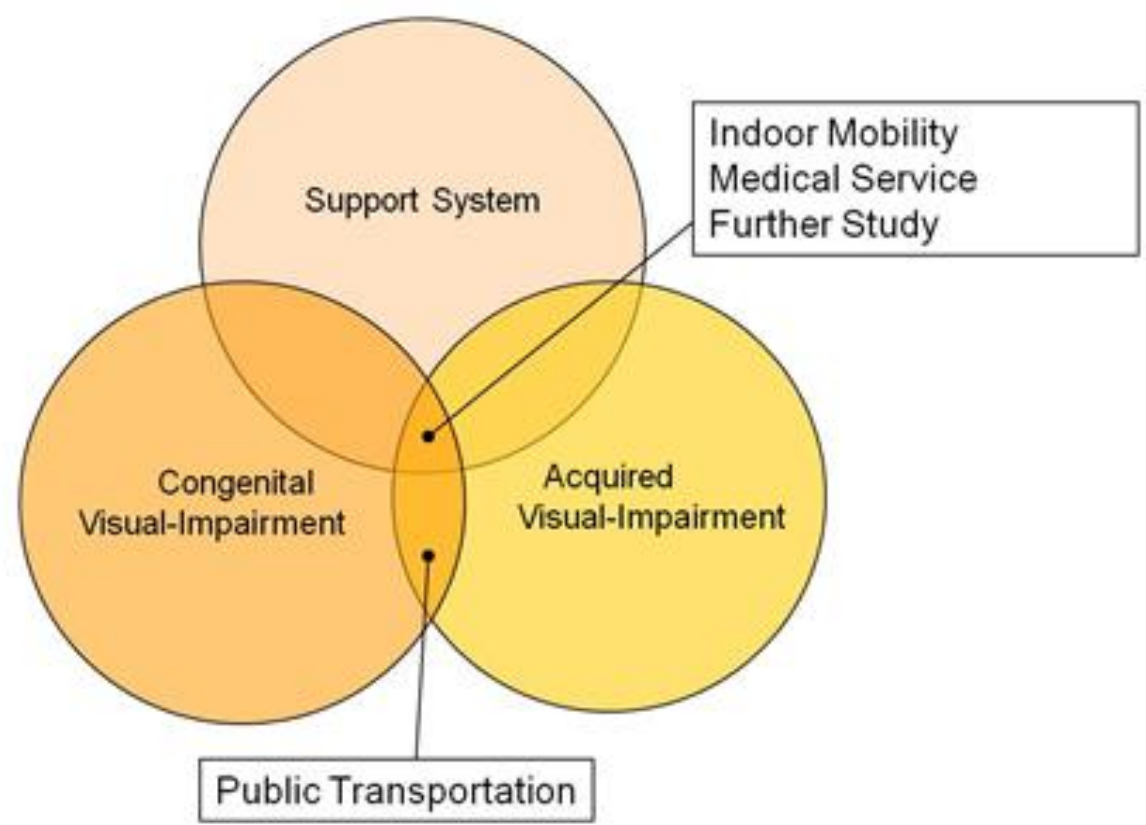

Fig. 5 Important but non-urgent. 


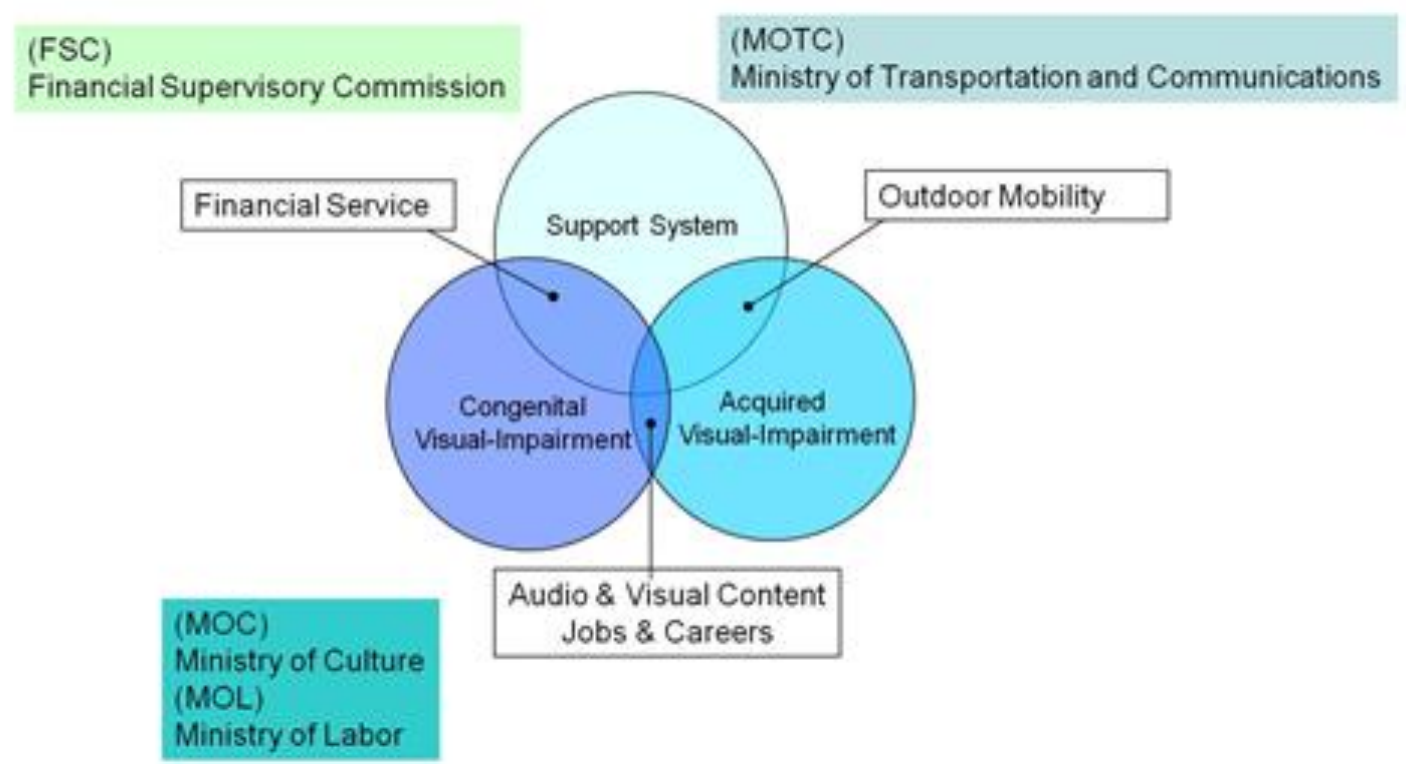

Fig. 6 Important and urgent.

People with acquired visual impairment consider outdoor mobility important and urgent. Because they are from sighted to sightless, they have desire in having a sense of freedom in doing outdoor activities. In this research, the most important items are those which both people with congenital or acquired visual impairment consider important and urgent. They are audio \& visual content and jobs \& careers. By comparing the services support systems provide now, the blind point is immediately shown. Services sighted people built may not be the real needs of visually impaired people. Therefore, we have to apply agent of real-time audio description service to strengthen accessible services which truly solve problems.

\section{Acknowledgements}

This study is conducted under the "III Innovative and Prospective Technologies Project" of the Institute for Information Industry which is subsidised by the Ministry of Economy Affairs of Taiwan.

\section{References}

[1] Kan, C. W. 2016. "Research on Cognitive Model of Visually Impaired User Adapt Audio Description Service: [The Survey on Visually-Impaired Users Need of Daily Life Tasks]." Innovative DigiTech-Enabled Applications \& Services Institute, Institute for Information Industry.

[2] Kan, C. W. 2014. Mobile Web Application Accessibility-Operation on Visually Impaired User Experience on Smart Devices APP, NCTU, I.I.M.

[3] Ezaki, N., Bulacu, M., and Schomaker, L. 2004, August. "Text Detection from Natural Scene Images: Towards a System for Visually Impaired Persons." In Pattern Recognition, 2004. ICPR 2004. Proceedings of the 17th International Conference 2: 683-6.

[4] Chen, X., and Yuille, A. L. 2004, June. "Detecting and Reading Text in Natural Scenes." In Computer Vision and Pattern Recognition, 2004. CVPR 2004. Proceedings of the 2004 IEEE Computer Society Conference 2: II-366.

[5] Arksey, H., and Knight, P. 1999. Interviewing for Social Scientists: An Introductory Resource with Examples. Thousand Oaks, CA: Sage.

[6] Coffey, A., and Atkinson, P. 1996. Making Sense of Qualitative Data. Thousand Oaks, CA: Sage. 